\title{
FPGA Implementation of Real Time Data Acquisition System Using Micro blaze Processor.
}

\author{
D.Sathish kumar \\ CVR College of Engineering \\ EIE Department \\ Hyderabad, India
}

R.Ganesh

CVR College of Engineering

ECE Department

Hyderabad, India

\begin{abstract}
The Progression of the human existence from the primitive state to the present technological complex state is just an outcome of observation of this environment. By observing the environment and controlling the various physical parameters like temperature, pressure of the environment we are able to sustain on this beautiful earth. In order to control physical parameters there is a need of a specific system called Data Acquisition System (DAS). Man has developed many different Data Acquisition Systems like Microprocessor based, PLC based, from Rock world to the Rocket world. This paper describes the implementation of Data Acquisition Systems using Embedded Processors.
\end{abstract}

Keywords - Data Acquisition, Virtex 5 FPGA Board, Micro blaze, GPIO, RTD Transducer

\section{INTRODUCTION}

In the real world all the physical quantities are continuous and measurable in analog domain, whereas in the electronic world of microprocessors, data storage, and automation is a digital domain. The conversion of parameters from analog domain to digital domain is the major advancement in semiconductor world with improvement in sensitivity, speed of conversion and parallel processing.

Data acquisition is the process of measuring quantities in the analog domain and converting it into the digital realm where any digital device can understand, record, and process and provides corresponding output in analog domain. The output could be visual, audible or any other electrical signal. The traditional method of data acquisition was to access analog data from field level and generates corresponding control action using comparators or any other analog circuitry.

This paper describes a way to design and implement a high performance and parameterized DAS on a single Virtex5 FPGA, which has more flexibility, power-efficiency, reconfigurabilty. The proposed DAS design can be implemented by using Xilinx Embedded Development Kit (EDK) ver. 12.4.

\section{DATA ACQUISITION SYSTEM}

Data acquisition products serve as a focal point in a system, tying together a wide variety of products, such as sensors that indicate temperature, flow, level, or pressure.

A digital data acquisition system includes the blocks shown in Figure.1.The essential operations of data acquisition system are:

1. Handling analog signals

2. Measurement

3. Data conversion

4. Programming and control

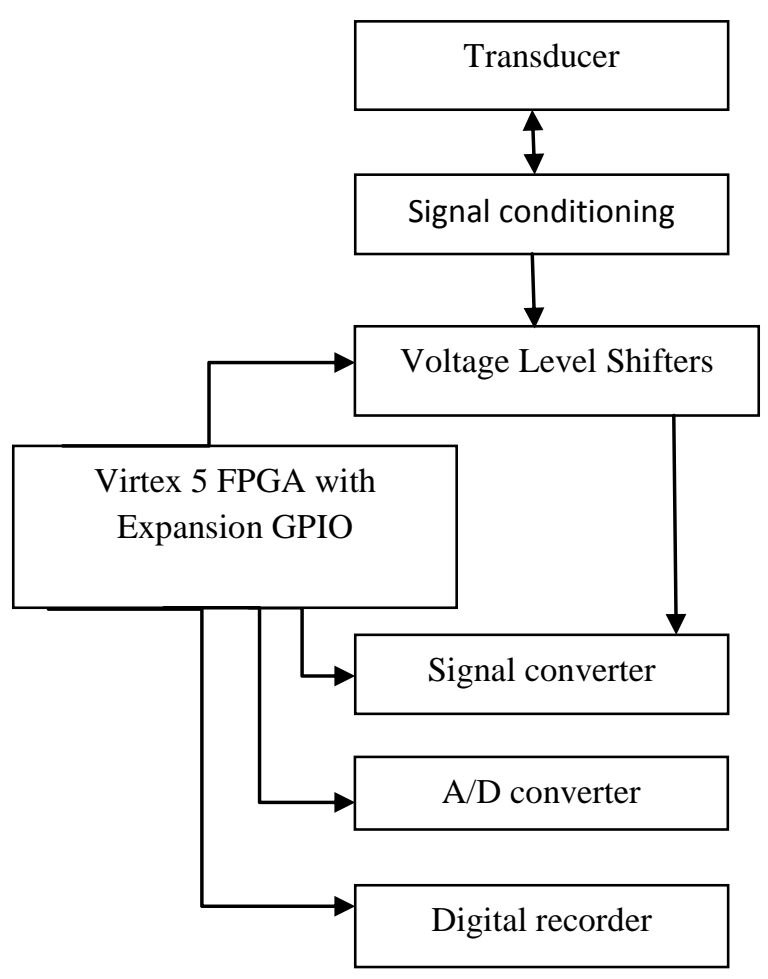

Figure 1. Block diagram of FPGA - DAS

The process of data acquisition is a step by step process, starting from sensing or collecting stimuli from external source or primary sensor to the final stage of proper alignment and storage of data in specific time duration. 


\section{COMPONENTS OF DATA ACQUISTION}

The following are the different blocks or components of Data acquisition system:
1. Transducers
2. Signal conditioning circuits
3. Voltage translators
4. Analog to Digital converter
5. Microblaze
6. GPIO
7. Digital recorders

\subsection{Transducers}

They convert a physical quantity into an electrical signal which is acceptable by data acquisition system.

For eg: Platinum RTD which functions on based on positive temperature coefficient principle can be used to acquire the temperature.

$$
\mathrm{R}_{\mathrm{T}}=\mathrm{R}_{\mathrm{o}}\left[1+\alpha_{\mathrm{o}}\left(\mathrm{T}-\mathrm{T}_{\mathrm{o}}\right)\right] \text {; where Rt is the RTD resistance }
$$

for change in temperature.

\subsection{Signal conditioning circuit}

The signal conditioning is essential as the transducer electrical output may be in micro or milli volts. Proper signal conditioning circuitry can be selected depending on transducer. Fr eg RTD can be connected to a Wheatstone bridge, Capacitive Transducer can be connected to a OP Amp circuit having a rich gain factor $(\beta)$. The strengthened voltage can be connected to next phase of DAS.

\subsection{Voltage Translator}

The toughest part of DAS is configuring the voltage levels of the FPGA with Real time devices. The FPGA contains expansion headers on board, which are called as GPIO slots. (In addition GPIO soft IP is also presented in EDK tool.) Through these Expansion headers the real time devices like ADC; DAC can be connected in order to acquire physical data by the Microblaze processor of FPGA. Hence there will be a requirement of voltage adjustments between FPGA and outside ADC chip. Virtex 5FPGA supports $3.3 \mathrm{~V}$ signals, ADC delivers $5 \mathrm{~V}$ signals. Appropriate signal conditioning level shifters have to be selected to overcome this typical phase of DAS Implementation.

\subsection{Analog to Digital Converter}

An A/D converter e.g.: ADC0809 is a data acquisition successive approximate component is a monolithic CMOS device with an 8 bit digital out, 8 channel multiplexer, and microprocessor compatible logic. The working of ADC can be programmed by the timing signal study where its initiation and control signals have to program by GPIO of Micro blaze in Virtex 5.

\subsection{Micro blaze}

The Micro blaze is a soft processor core designed for XilinxFPGAs from Xilinx. The Embedded development kit [5], [6] is used to implement hardware design consisting IP cores and a Microblaze Soft Processor. The DAS design is implemented by writing the ADC logic in EDK software application to run on the Microblaze processor. The software application controls the functionality of different IP cores added to the processor. SystemC [4] programming language is used for developing software application

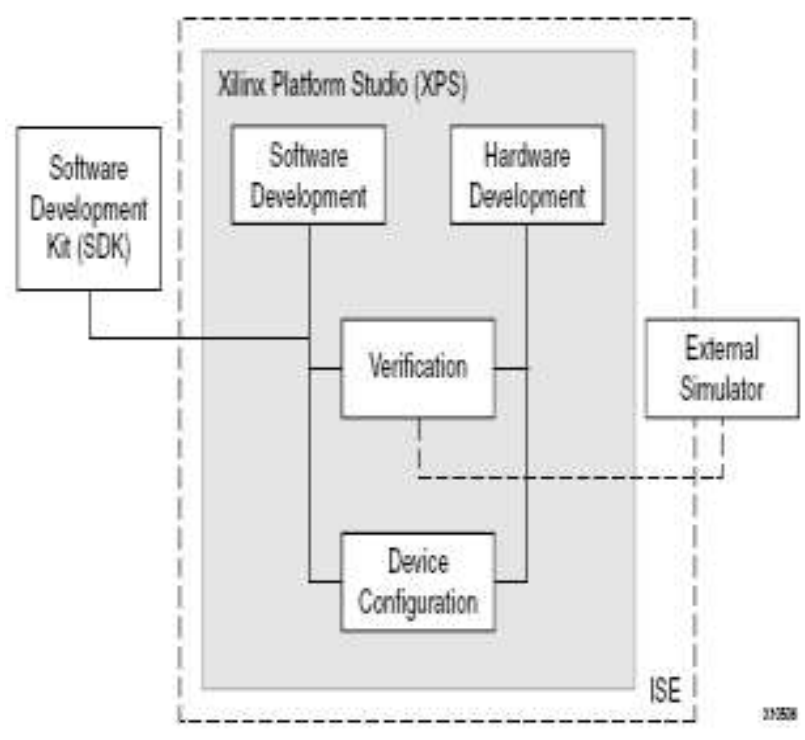

Figure 2. EDK tool flow block dig

\subsection{GPIO}

A range of applications can be developed with Microblaze processor. The applications may range from a simple state machine to Full custom Embedded system. DAS is a full custom Embedded System, hence it uses UART, GPIO, Timer \& Interrupt controller, External memory along with Microblaze , The expansion headers provided on FPGA board can be configured as a channel of communication between GPIO (target to Microblaze) and ADC 0809. EDK 12.4 tool helps to provide the communication between the master and slave by using Base system Builder (BSB) [5]

\subsection{Digital Recorders}

These records the data obtained by the Microblaze in nonvolatile memory for future reference. Normally UART can be used as a soft IP for recording the values. This paper implements the digital display of equivalent temperature obtained by the Transducer under supervision of Microblaze.

\section{IMPLEMENTATION USING MICROBLAZE}

The implementation of data acquisition system include

$>$ Micro Blaze

$>$ User machine interface

\section{A. Micro blaze}

Shared memory

Micro blaze is a 32 bit RISC Harward architecture soft core processor with advanced architecture options i.e., PLB interface, memory management unit, instruction and data-side cache, configurable pipeline depth, floating point unit, etc., .it has over 70-user configurable options, enabling virtually any processor use case from a very small footprint microcontroller 
to a high performance computive-intensive system running Linux.[5]

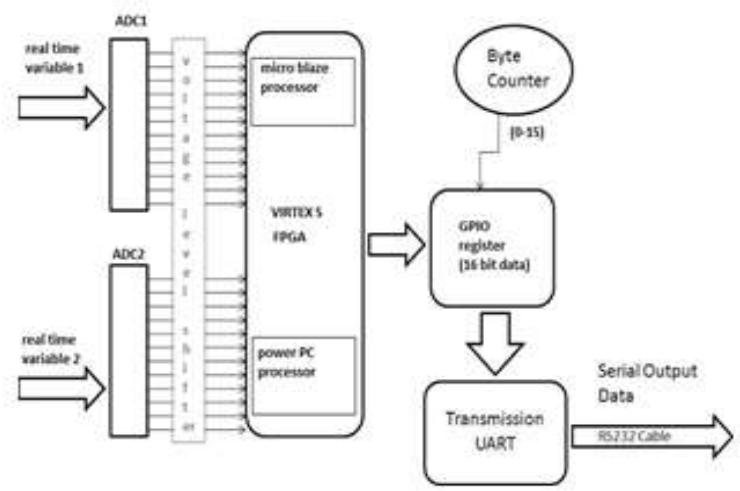

Figure 3. Flow Diagram for FPGA

\section{B .User Machine Interface}

The physical interface between FPGA and ADC is made through a GPIO peripheral IP. A timer peripheral IP is added to the project to allow the MicroBlaze to measure time delays. A delay function will be used to create the required signal timing for the ADC interface.

\section{Shared Memory}

A Dual Port Block Random Access Memory (DP-BRAM) is the memory used to store the values of coefficients computed by Microblaze processor. Block RAM (BRAM) IP Block and XPS BRAM Controller IP are added to the Microblaze system to implement Dual Port BRAM.

\section{Process Description}

The temperatures from two different analog sensors i.e., RTD are converted to digital form using $\mathrm{ADC}$ and the latter is fed to Microblaze Processor operating at $125 \mathrm{MHz}$ through voltage translators. To make proper communication with ADC, the Microblaze processor utilizes General Purpose Input Output (GPIO) soft IP in a programmed way for sending the signals from Microblaze and vice versa. The ADC is initiated by one of the data received by the FPGA is in digital format, which is processed and the temperature is displayed on screen. Here we can observe two different parameters are measured simultaneously.

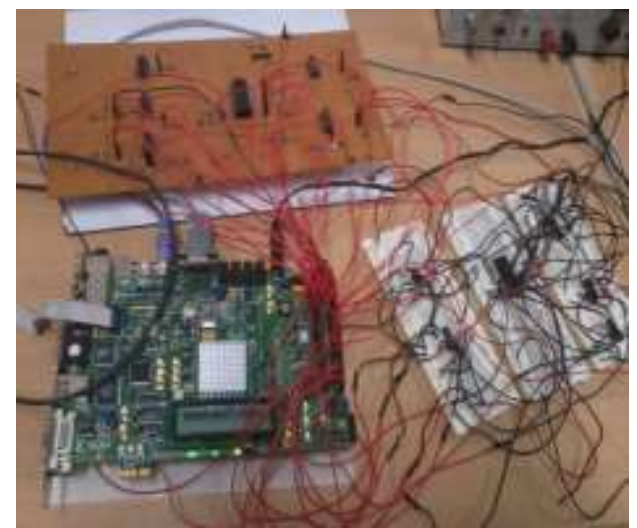

Figure 4. User machine interface

\section{RESULTS}

The results obtained by the Implementation of DAS using FPGA are provided in the digital format on the hexadecimal scale which can be converted into decimal format and displayed by HyperTerminal. The Table.1 gives the list of temperatures and their corresponding voltage outputs collected on HyperTerminal.

\begin{tabular}{|c|c|c|c|}
\hline \multirow[t]{2}{*}{ Temperature } & \multirow{2}{*}{$\begin{array}{l}\text { Voltage } \\
\text { (V) }\end{array}$} & \multicolumn{2}{|c|}{ HyperTerminal value } \\
\hline & & E.O.C & D7-D0 \\
\hline $61^{\circ} \mathrm{C}$ & 3.83 & 1 & $\mathrm{CC}$ \\
\hline $64^{\circ} \mathrm{C}$ & 3.88 & 1 & D0 \\
\hline $67^{\circ} \mathrm{C}$ & 3.91 & 1 & D4 \\
\hline $70^{\circ} \mathrm{C}$ & 3.93 & 1 & D8 \\
\hline $73^{\circ} \mathrm{C}$ & 3.95 & 1 & $\mathrm{DC}$ \\
\hline $76^{\circ} \mathrm{C}$ & 3.96 & 1 & E0 \\
\hline $79^{\circ} \mathrm{C}$ & 3.98 & 1 & $\mathrm{E} 4$ \\
\hline $82^{\circ} \mathrm{C}$ & 3.99 & 1 & E8 \\
\hline $85^{\circ} \mathrm{C}$ & 4.01 & 1 & $\mathrm{EC}$ \\
\hline $88^{\circ} \mathrm{C}$ & 4.21 & 1 & F0 \\
\hline $91^{\circ} \mathrm{C}$ & 4.52 & 1 & $\mathrm{~F} 4$ \\
\hline $94^{\circ} \mathrm{C}$ & 4.72 & 1 & F8 \\
\hline $97^{\circ} \mathrm{C}$ & 5.03 & 1 & $\mathrm{FE}$ \\
\hline
\end{tabular}

Table.1 Temperature and Voltage output values

This data can be stored in a mass storage device like USB which can be designed as a soft IP on FPGA

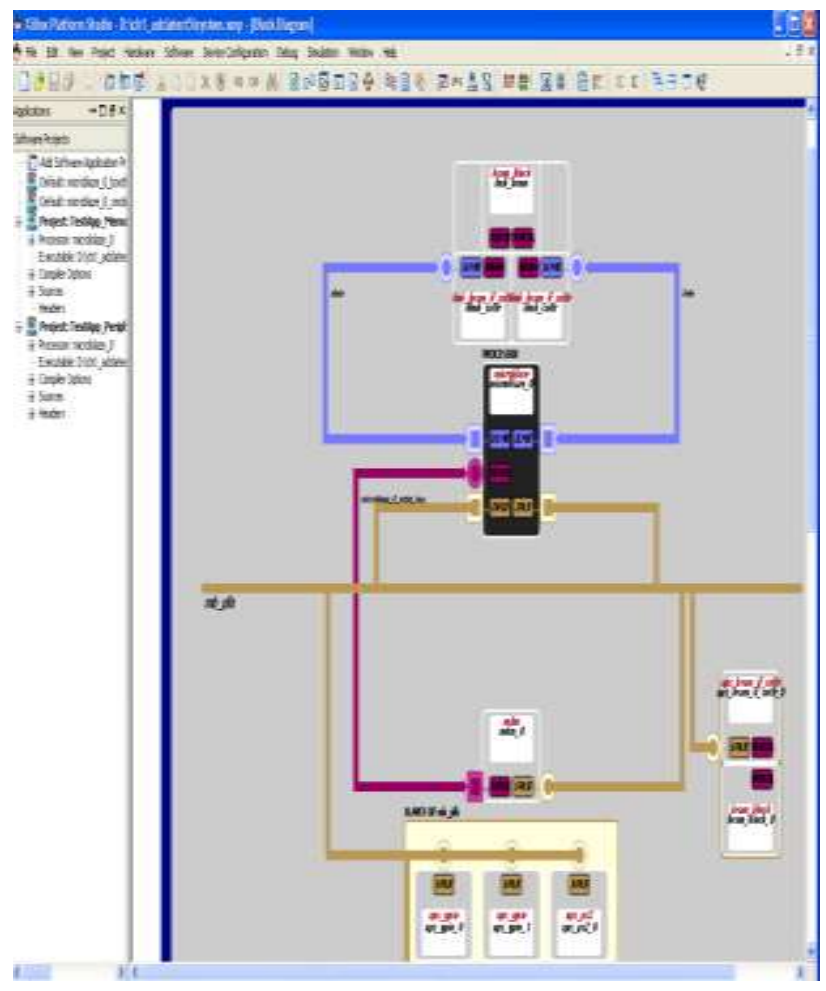

Figure 5 Block Diagram of Micro blaze Sub module 


\section{CONCLUSION}

The Microblaze processor being a soft-core processor is added advantage compared to a hard processor which in turn is costly in terms of reconfiguring it. The programmable input output lines made it to interface with many of the real-time applications with the only cost of voltage translators or level shifters. The high speed clock of soft processor improves the speed of execution, the added advantage.

The programmable input output lines of FPGA can be used to interface more number of digital devices and the slots can also be expanded in order to increase further more number of digital devices attached. Where for the other type of processors offers limited connectivity.

\section{REFERENCES}

[1] Embedded systems tool reference manual" EDK 12.4 version.

[2] Shebli Anvar, Olivier gachelin, Pierre Kestener, Herve Le Provost, Irakli Mandjavidze,"FPGA - based System-onchip Designs for Real-Time Applications in Particle Physics", $14^{\text {th }}$ IEEE Real-Time Conference, Stockholm, Sweden, June 6-10, 2005.

[3] S. Thanee S. Somkuarnpandit and K. Saetang," FPGA Based Multi Protocal data Acquisition System with High Speed USB Interface".

[4] "Microblaze Processor Reference Guide", Embedded Development Kit EDK 12.4 version.

[5] A. Sagahyroon,T.Al-kudairi, "FPGA Based Acquistion of sensor Data",InternationalConference on Information. and Communication Technology, ,ICTTA 2006.

[6] http:/www.xilinx.com/tools/microblaze.html

[7] "Microblaze Processor Reference Guide" 